\title{
An Active Viewing Framework for Video-Based Learning
}

\author{
Samuel Dodson \\ University of British Columbia \\ dodsons@mail.ubc.ca
}

\author{
Dongwook Yoon \\ University of British Columbia \\ yoon@cs.ubc.ca
}

\author{
Ido Roll \\ University of British Columbia \\ ido.roll@ubc.ca
}

\author{
Negar M. Harandi \\ University of British Columbia \\ negarm@ece.ubc.ca
}

\author{
Matthew Fong \\ University of British Columbia \\ mfong@ece.ubc.ca
}

\author{
Sidney Fels \\ University of British Columbia \\ ssfels@ece.ubc.ca
}

\begin{abstract}
Video-based learning is most effective when students are engaged with video content; however, the literature has yet to identify students' viewing behaviors and ground them in theory. This paper addresses this need by introducing a framework of active viewing, which is situated in an established model of active learning to describe students' behaviors while learning from video. We conducted a field study with 460 undergraduates in an Applied Science course using a video player designed for active viewing to evaluate how students engage in passive and active video-based learning. The concept of active viewing, and the role of interactive, constructive, active, and passive behaviors in videobased learning, can be implemented in the design and evaluation of video players.
\end{abstract}

\section{Author Keywords}

active viewing, video-based learning, annotation

\section{INTRODUCTION}

Previous work has found that students' active engagement can improve their learning outcomes [10, 13], especially when compared with passive engagement $[2$, 8]. Active learners make use of affordances in learning objects and environments in order to control their learning. They regulate their learning in accordance with their knowledge and goals [11]. The concept of active learning has not yet been applied to video-based learning. With a framework of active viewing, we identify potential affordances for learning with video.

This paper introduces and operationalizes the concept of active viewing as follows. First, we introduce the concept and ground it in a constructivist view of learning. Second, we describe a study with a video player

Permission to make digital or hard copies of all or part of this work for personal or classroom use is granted without fee provided that copies are not made or distributed for profit or commercial advantage and that copies bear this notice and the full citation on the first page. Copyrights for components of this work owned by others than the author(s) must be honored. Abstracting with credit is permitted. To copy otherwise, or republish, to post on servers or to redistribute to lists, requires prior specific permission and/or a fee. Request permissions from permissions@ acm.org.

L@S 2018, June 26-28, 2018, London, United Kingdom

(C) 2018 Copyright held by the owner/author(s). Publication rights licensed to ACM ISBN 978-1-4503-5886-6/18/06. . \$15.00

DOI: https://doi.org/10.1145/3231644.3231682 that facilitates active viewing by supporting interaction with visual and textual representations of video content and personalization with highlights and notes. This study, which was conducted for seven weeks in an undergraduate Applied Science class of 460 students, provides in vivo data from students using video to prepare for lectures, homework, and exams. Using the data collected in our study, we describe how students use the video player affordances in order to take control of their learning. Third, we suggest that when provided with a video player designed for active viewing, students engage in different behaviors to support their learning. This paper provides a foundation for designing and evaluating video players to support videobased learning by filling a gap in the literature on students' engagement with video.

\section{ACTIVE VIEWING FRAMEWORK}

The current literature on video-based learning lacks sufficient understanding of how students' behaviors are related to their engagement with video content. Consequently, this paper works towards a framework of active viewing by describing students' behaviors using the ICAP framework [2], which distinguishes between the interactive, constructive, active, and passive categories of behaviors. Several video-based learning behaviors, and students' motivations for using them, are described in Table 1 . The behaviors were identified by reviewing the literature, drawing on active learning in other media, and from our observations of, and conversations with, students.

Active viewing includes interactive, constructive, and active behaviors, which treat video as a non-linear medium. These behaviors are described by Chi and Wylie [3]. Interactive behaviors involve collaborating, cooperating, and communicating with instructors and peers in video. The interactive category is based on a socio-constructive understanding of learning, which emphasizes learning as a socially-driven process. Several systems have supported peer-to-peer interactions with video content. Bargeron et al.'s MRAS offered temporally-anchored discussion threads between students [1]. Dorn et al.'s TrACE went a step further by supporting spatially-anchored discussion threads [5]. A 
Table 1. Behaviors of active and passive viewing, categorized using the ICAP framework.

\section{Interactive Behaviors}

Communicating with others.

Cooperating with others.

Collaborating with others.

Constructive Behaviors

Making connections between learning objects.

Taking notes to record sense making.

Highlighting video content for future use.

Tagging video content for future use.

\section{Active Behaviors}

Browsing for general information.

Searching for specific information.

Triaging between learning objects, such as a video and a textbook.

$R e$-watching specific video content.

Pausing video content to reflect on the video content or engage in another viewing behavior.

\section{Passive Behaviors}

Playing video content.

recent trend is to extract useful information by aggregating users' interactions with video content. For example, Glassman et al.'s Mudslide used user-generated highlights to identify areas of confusion or importance within videos [7]. Constructive behaviors, such as taking notes, are used to record meaning making. Through these activities, learners construct their own meaning in a way that expands and extends beyond the video itself. This also includes making connections between concepts. Active behaviors include, but are not limited to, browsing, searching, pausing, changing playback speed, and re-watching video content. Previous work suggests that annotating may support textbased learning processes and outcomes [9, 12]; however, there are few studies investigating whether or not annotating provides similar benefits when learning with video [4]. In contrast to these categories of behaviors that make up active viewing, passive viewing simply involves watching the video linearly, without interaction [3]

\section{FIELD STUDY OF VIDEO-BASED LEARNING}

To explore how students learn with video, we conducted a field study using ViDeX [6], a video player designed for active viewing, in an undergraduate Applied Science course of 460 students. Students use of ViDeX was logged for seven weeks and then analyzed. Students were provided with 14 optional videos $(8 \pm 3$ minutes) in which their instructor lectures over a deck of slides. During the seven-week study, 219 of the students $(48 \%)$ used ViDeX at least once. In this paper, we focus on their interactions with ViDeX, acknowledging that active learning can also use additional resources, such as paper or electronic notebooks. The log data were supplemented by an online questionnaire. The 112 respondents were asked about their video-

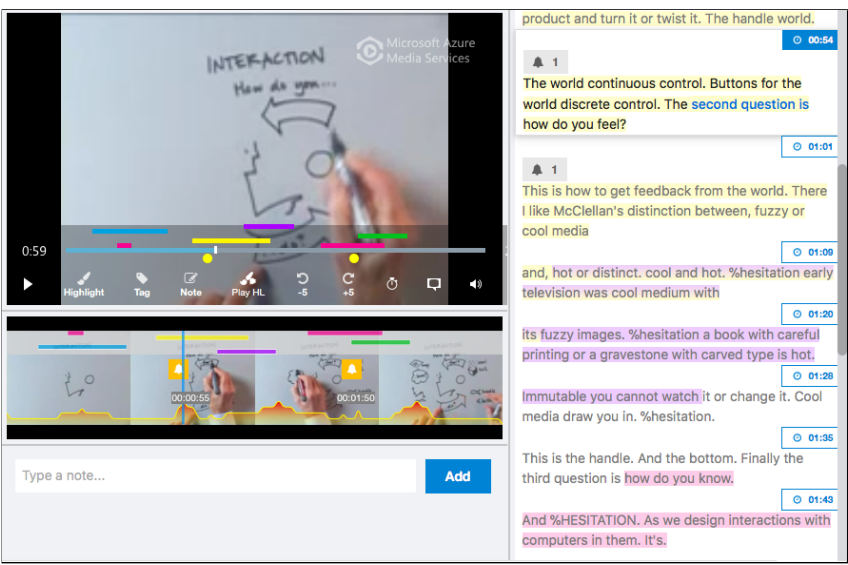

Figure 1. ViDeX with student-generated highlights and tags. The Player is displayed at the top left, the Filmstrip at the middle left, the note taking area at the bottom left, and the Transcript at the right. Students can select intervals for highlighting using either the Filmstrip or the Transcript. At the bottom of the Filmstrip, a histogram displays the amount of times a student has watched specific parts of the video.

based learning practices, including how and why they highlighted and took notes using ViDeX. Students were not compensated for participating in the study.

\section{ViDeX: A Video Player for Active Viewing}

To fully support video-based learning, video players should provide tools that go beyond traditional playback features. Unfortunately, the majority of video viewers only support passive and active behaviors, which are not as effective for learning as constructive and interactive behaviors. The version of ViDeX used in this study was designed to support a broader set of behaviors, as described in Table 1, than most video players. ViDeX has four main components: the Player, the Filmstrip, the Transcript, and the note taking area, as shown in Figure 1.

Students can use ViDeX to interact with and personalize video content. The Filmstrip and Transcript provide students with visual and textual representations of the video content, respectively. The Filmstrip supports visual navigation of video content through thumbnails. Students can hover their cursor over a thumbnail to preview the visual content of a specific interval of video content. The Transcript enables navigation of the textual content of a video. The Transcript allows students to read ahead or behind the playhead. A tell-how video, with much narration and little demonstration, results in a useful Transcript and a less useful Filmstrip, whereas a show-how video, with much demonstration and little narration, results in a useful Filmstrip and a less useful Transcript.

To further support active viewing behaviors, ViDeX allows for highlighting and note taking. After clicking on and dragging across either the Filmstrip or the Transcript to select an interval of visual or textual content, 
students can choose a color to highlight the selected interval. If students would like to make annotations that are more verbose than a highlight, they can write notes that are then linked to a specific time in the video.

\section{Results}

We analyzed the ViDeX log data and found that students' most frequent behaviors aligned with our definition of passive and active viewing: i) all participants played one or more videos; ii) the playback speed was changed by 60 participants $(27 \%)$ on average of 10 times each; and iii) 139 participants (63\%) re-watched one or more videos on average 12 times each (see Table 2).

Students also regularly engaged in constructive behaviors. Forty participants (18\%) highlighted, creating 333 highlights in total. The highlighting behaviors of participants were idiosyncratic, and highlights varied in color, temporal length, and positions within the videos. We asked the survey respondents about the ease of use and usefulness of highlighting with ViDeX. Fifty-four percent $(54 \%)$ of respondents agreed or strongly agreed that highlighting was easy, while $7 \%$ disagreed or strongly disagreed. Forty-three percent (43\%) agreed or strongly agreed that highlighting was useful, while $20 \%$ disagreed or strongly disagreed.

The highlights may have been used to support other active viewing behaviors. Sixty-seven percent (67\%) of highlights were the destination of a seek, suggesting that highlights helped participants re-watch specific parts of video. The number of highlights directly sought is likely greater than $67 \%$, as we only counted a seek when a user clicked within the span of a highlight. Of the highlights that were sought to, the median number of times they were sought to was two $(M=3.2, S D=3.8)$, suggesting that participants revisited confusing or important parts of videos multiple times. The temporal length of highlights that were sought to were larger than those that were not re-watched (median $=7.5$ seconds and median $=5.6$ seconds, respectively). A Welch-Two-Sample t-test found that this difference is statistically significant $(t=3.102, d f=197.12, p=.002)$.

Twenty-six (12\%) participants created one or more notes, creating 83 notes in total. The median note length was 21 words $(M=31.0, S D=28.5)$. Fifteen of the 40 participants that highlighted (38\%) also took one or more notes. Fifty percent (50\%) of survey respondents agreed or strongly agreed that note taking with ViDeX was easy, while $18 \%$ disagreed or strongly disagreed. Thirty-nine percent (39\%) of participants agreed or strongly agreed that note taking was useful, while $25 \%$ disagreed or strongly disagreed.

To assess whether or not there was a difference between highlighting and note taking, we compared these results with those reported earlier on highlighting. McNemar's test was used to determine if there was a dif-
Table 2. Logged behaviors, including the percentage of students that engaged in each behavior and the mean number of times they did so. Interactive behaviors are not reported on in this paper, as design of the system did not yet specifically support cooperation, collaboration, and communication at the time of data collection.

\begin{tabular}{lcc}
\hline Behavior & $\%$ of Students & Mean Occurrences \\
\hline $\begin{array}{l}\text { Passive } \\
\text { Watching Video }\end{array}$ & $100 \%$ & 74 times each \\
Active & & 10 times each \\
Changing Play- & $27 \%$ & \\
back Speed & & 12 times each \\
$\begin{array}{l}\text { Re-watching } \\
\text { Pausing }\end{array}$ & $63 \%$ & 71 times each \\
Constructive & $97 \%$ & 8 times each \\
Highlighting & $18 \%$ & 3 times each \\
Note Taking & $12 \%$ & \\
\hline
\end{tabular}

ference in the ease of use of highlighting and note taking $(p=.112)$ and the usefulness of highlighting and note taking $(p=.721)$; however, no statistically significant differences were found.

\section{DISCUSSION}

The results suggest that students make use of video player affordances for active viewing. Participants regularly engaged in active and constructive behaviors. It is important not to label students as active or passive viewers based on the frequency of their behaviors alone. Passive behaviors can, for example, support interactive, constructive, and active behaviors. Consequently, students often engage in active and passive viewing behaviors when learning from video. We suspect that students are using these behaviors for multiple reasons. They may try to maximize efficiency by minimizing the amount of time they spend watching videos. Students may also try to personalize the video content to their needs. Active viewing is used by students to make decisions about which parts of the video they need to watch.

ViDeX provides visual and textual representations of video content - visual in the form of the Filmstrip and the Player and textual in the form of the Transcript. We found that students highlight mainly using the Transcript, possibly because they highlight textual, tell-how information or because students may be used to highlighting text. Instances of highlighting, using either the Filmstrip or the Transcript, and subsequent seeking to the highlighted video content using the other interface was common. This suggests that students were able to use the Filmstrip and the Transcript selectively. We anticipate that over time, students will develop strategies that take advantage of the affordances provided by different interfaces and representations of video content. Additional research is needed to fully explore how these new strategies emerge as students develop active viewing practices. 


\section{CONCLUSION}

This paper describes the beginning of an active viewing framework, grounded in the ICAP framework. As video players are re-designed to better support active viewing, new affordances for interactive, constructive, and active behaviors are a priority. We believe students will make use of these affordances to suit their style of learning and preferences, similar to how some students annotate their textbooks liberally while others do not. Furthermore, as students make use of active viewing affordances in video players, they will develop new strategies to support their video-based learning which will, in turn, lead to new behaviors that can be added to our active viewing framework. Taking this a step further, video content may then be re-imagined with the aim of creating more active experiences of engaging with video.

\section{Future Work}

Our analysis treated instances of constructive, active, and passive viewing behaviors as independent of each other. Future work could investigate sequences of behaviors, or video-based learning strategies. For example, what behaviors do students engage in before and after highlighting? Identifying common video-based learning strategies could then further inform the design of educational video players with the affordances students need to effectively study from video. Methods for measuring learning processes and outcomes in relation to active viewing also need to be investigated.

Video is used in addition to, and sometimes in place of, traditional lectures, introducing a paradigm shift that fundamentally changes the way instructors teach and students learn. By providing mechanisms that make use of familiar text-based interactions and apply them to video, as well as create completely novel affordances tuned to the unique properties of videos, we have the opportunity to elevate video-based learning to be an interactive medium. By studying active viewing, we hope to better understand video-based learning and design video viewers to help students fully realize the potential of video as a medium for learning.

\section{Acknowledgements}

This work was funded by the University of British Columbia Teaching and Learning Enhancement Fund, Natural Sciences and Engineering Research Council of Canada and Microsoft Corporation.

\section{REFERENCES}

1. David Bargeron, Jonathan Grudin, Anoop Gupta, Elizabeth Sanocki, Francis Li, and Scott Leetiernan. 2002. Asynchronous collaboration around multimedia applied to on-demand education. Journal of Management Information Systems 18, 4 (2002), 117-145.

2. Michelene T H Chi. 2009. Active-constructive-interactive: A conceptual framework for differentiating learning activities. Topics in Cognitive Science 1, 1 (2009), 73-105.
3. Michelene T H Chi and Ruth Wylie. 2014. The ICAP framework: Linking cognitive engagement to active learning outcomes. Educational Psychologist 49, 4 (2014), 219-243.

4. Samuel Dodson, Ido Roll, Matthew Fong, Dongwook Yoon, Negar M. Harandi, and Sidney Fels. 2018. Active viewing: A study of video highlighting in the classroom. In Proceedings of the 2018 Conference on Human Information Interaction \& Retrieval. ACM, New York, NY, 237-240.

5. Brian Dorn, Larissa B Schroeder, and Adam Stankiewicz. 2015. Piloting TrACE: Exploring spatiotemporal anchored collaboration in asynchronous learning. In Proceedings of the 18th ACM Conference on Computer Supported Cooperative Work 85 Social Computing. ACM, New York, NY, 393-403.

6. Matthew Fong, Samuel Dodson, Xueqin Zhang, Ido Roll, and Sidney Fels. 2018. ViDeX: A platform for personalizing educational videos. In Proceedings of the 18th ACM/IEEE Joint Conference on Digital Libraries. ACM, New York, NY, 331-332.

7. Elena L Glassman, Juho Kim, Andrés Monroy-Hernández, and Meredith Ringel Morris. 2015. Mudslide: A spatially anchored census of student confusion for online lecture videos. In Proceedings of the 33rd Annual ACM Conference on Human Factors in Computing Systems. ACM, New York, NY, 1555-1564.

8. Richard R Hake. 1998. Interactive-engagement versus traditional methods: A six-thousand-student survey of mechanics test data for introductory physics courses. American Journal of Physics 66, 1 (1998), 64-74.

9. Catherine C Marshall. 1998. Toward an ecology of hypertext annotation. In Proceedings of the Ninth ACM Conference on Hypertext and Hypermedia. ACM, New York, NY, 40-49.

10. Michael Prince. 2004. Does active learning work? A review of the research. Journal of Engineering Education 93, 3 (2004), 223-231.

11. Ido Roll and Philip H Winne. 2015. Understanding, evaluating, and supporting self-regulated learning using learning analytics. Journal of Learning Analytics 2, 1 (2015), 7-12.

12. Michele L Simpson and Sherrie L Nist. 1990. Textbook annotation: an effective and efficient study strategy for college students. Journal of Reading 34, 2 (1990), 122-129.

13. Dongsong Zhang, Lina Zhou, Robert O Briggs, and Jay F Nunamaker. 2006. Instructional video in e-learning: Assessing the impact of interactive video on learning effectiveness. Information \& Management 43, 1 (2006), 15-27. 